\title{
Ultrafast science and development at the Artemis facility
}

\author{
I. C. Edmond Turcu ${ }^{1}$, Emma Springate ${ }^{1}$, Chris A. Froud ${ }^{1}$, Cephise M. Cacho ${ }^{1}$, John L. Collier ${ }^{1,2}$, \\ William A. Bryan ${ }^{1,2}$, G.R.A. Jamie Nemeth ${ }^{1,2}$, Jon P. Marangos ${ }^{3}$, John W.G. Tisch ${ }^{3}$, \\ Ricardo Torres $^{3}$, Thomas Siegel ${ }^{3}$, Leonardo Brugnera ${ }^{3}$, Jonathan G. Underwood ${ }^{1,4}$, \\ Immacolata Procino ${ }^{4}$, W. Roy Newell ${ }^{4}$, Carlo Altucci ${ }^{5}$, Raffaele Velotta ${ }^{5}$, Raymond B. King ${ }^{6}$, \\ John D. Alexander ${ }^{6}$, Chris R. Calvert ${ }^{6}$, Orla Kelly ${ }^{6}$, Jason B. Greenwood ${ }^{6}$, Ian D. Williams ${ }^{6}$, \\ Andrea Cavalleri ${ }^{7,8}$, Jesse C. Petersen ${ }^{7,8}$, Nicky Dean ${ }^{7}$, Sarnjeet S. Dhesi ${ }^{9}$, Luca Poletto ${ }^{10}$, \\ Paolo Villoresi ${ }^{10,11}$, Fabio Frassetto ${ }^{10}$, Stefano Bonora ${ }^{10}$, Mark D. Roper ${ }^{12}$. \\ ${ }^{1}$ Central Laser Facility, STFC Rutherford Appleton Laboratory, UK. ${ }^{2}$ Department of Physics, \\ Swansea University, UK. ${ }^{3}$ Blackett Laboratory, Imperial College London, UK. ${ }^{4}$ Department of \\ Physics and Astronomy, University College London, UK. ${ }^{5}$ CNSIM and Dipartimento di Scienze \\ Fisiche, Universita di Napoli 'Federico II', Naples, Italy. ${ }^{6}$ School of Mathematics and Physics, \\ Queen's University Belfast, UK. ${ }^{7}$ Department of Physics, Clarendon Laboratory, University of \\ Oxford, UK. ${ }^{8}$ Max Planck Research Group for Structural Dynamics, Centre for Free Electron Laser \\ Science and University of Hamburg, Germany. ${ }^{9}$ Diamond Light Source, UK. ${ }^{10}$ LUXOR, CNR- \\ INFM, Padova, Italy. ${ }^{11}$ DEI-University of Padova, Italy. ${ }^{12}$ STFC Daresbury Laboratory, UK.
}

\begin{abstract}
The Artemis facility for ultrafast XUV science is constructed around a high average power carrier-envelope phasestabilised system, which is used to generate tuneable pulses across a wavelength range spanning the UV to the far infrared, few-cycle pulses at $800 \mathrm{~nm}$ and short pulses of XUV radiation produced through high harmonic generation. The XUV pulses can be delivered to interaction stations for materials science and atomic and molecular physics and chemistry through two vacuum beamlines for broadband XUV or narrow-band tuneable XUV pulses. The novel XUV monochromator provides bandwidth selection and tunability while preserving the pulse duration to within 10 fs. Measurements of the XUV pulse duration using an XUV-pump IR-probe technique demonstrate that the XUV pulselength is below $30 \mathrm{fs}$ for a 28 fs drive laser pulse. The materials science station, which contains a hemispherical electron analyser and five-axis manipulator cooled to $14 \mathrm{~K}$, is optimised for photoemission experiments with the XUV. The end-station for atomic and molecular physics and chemistry includes a velocity-map imaging detector and molecular beam source for gas-phase experiments. The facility is now fully operational and open to UK and European users for twenty weeks per year. Some of the key new scientific results obtained on the facility include: the extension of HHG imaging spectroscopy to the mid-infrared; a technique for enhancing the conversion efficiency of the XUV by combining two laser fields with non-harmonically related wavelengths; and observation of $\mathrm{D}_{3}{ }^{+}$photodissociation in intense laser fields.
\end{abstract}

Keywords: Ultrafast, femtosecond, few-cycle, high harmonic generation, XUV, carrier-envelope phase, photodissociation, time-resolved, electronic structure, electron dynamics. 


\section{INTRODUCTION}

Recent advances in the field of ultrafast laser technology are driving progress in our understanding of the response of matter to light on femtosecond to attosecond timescales ${ }^{1,2}$. The ability to lock the phase of the carrier frequency of a laser to the envelope of the pulse ${ }^{3}$ (carrier-envelope phase stabilisation, CEP) enables the generation of precisely controlled optical electric fields. The availability of laser pulses just a few optical cycles long ${ }^{4}$ has pushed the time resolution available at optical wavelengths to a few femtoseconds, fast enough to track the motion of protons. The combination of these two techniques means we can now generate few-cycle pulses whose electric field can be tailored through CEP control to, for example, give just one strong peak in the electric field. This has been used to control electron localisation in the dissociation of small molecules ${ }^{5}$ and for the generation of isolated attosecond pulses ${ }^{3}$. The availability of tuneable, femtosecond pulses at wavelengths spanning the ultraviolet to the infrared is also having an impact. In the past, most studies of strong field interactions on an ultrafast timescale have been at $800 \mathrm{~nm}$, restricted by the gain spectrum of Ti:Sapphire based systems. For example, changing the wavelength to the 1.2-1.6 micron region strongly changes the interaction as the ponderomotive energy that an electron gains in the laser field scales as the square of the wavelength ${ }^{6}$. As laser technology moves to higher repetition rates, the feasibility of using XUV pulses in the 10-100 eV energy range created through high harmonic generation increases. The shot-to-shot stability of the harmonics improves and the average photon flux increases to the point where complex experiments, such as photoemission measurements ${ }^{7}$, requiring averaging over many thousands of laser shots become possible.

The Artemis facility for ultrafast XUV science at the Central Laser Facility in the UK aims to capitalise on these advances by bringing them together in combination. The facility provides few-cycle, carrier-envelope phase stabilised and tuneable laser beamlines, XUV beamlines and interaction stations for gas-phase and condensed matter experiments. One key goal is to make XUV pulses available for applications, by combining expertise and technologies from the domains of femtosecond lasers and synchrotron science. The narrow-bandwidth, short XUV pulses provided through the monochromatised beamline on Artemis will allow time-resolved selective studies of atomic and molecular electronic states, offering better spectral resolution than attosecond pulses and better temporal resolution than a synchrotron. The facility is now fully operational and open to UK and European users to perform experiments using either the Artemis end-stations or their own.

In this paper, we first provide a detailed technical description of the Artemis facility, including the laser and XUV beamlines and the end-stations for materials science and atomic and molecular physics and chemistry. A novel measurement of the duration of XUV pulses exiting a pulse-length preserving monochromator is described, confirming that the monochromator can indeed preserve the time duration of the XUV pulses to below 30 fs. We then briefly describe some of the key new scientific results obtained on the facility, including; the observation of $\mathrm{D}_{3}^{+}$ photodissociation; the extension of HHG imaging spectroscopy to the mid-infrared and a technique for enhancing the conversion efficiency of the XUV by combining two laser fields with different, non-harmonically related wavelengths.

\section{THE ARTEMIS FACILITY}

Artemis is constructed around a carrier-envelope phase (CEP) stabilised femtosecond laser system. This can be used to generate tuneable pulses across a wavelength range spanning the UV to the far infrared, few-cycle pulses at $800 \mathrm{~m}$ and short pulses of XUV radiation through high harmonic generation (HHG). The XUV can be delivered to interaction stations for materials science and atomic and molecular physics through vacuum beamlines for broadband or monochromatic (narrowband) XUV pulses. An engineering drawing of the facility is shown in Figure 1.

\subsection{Laser beamlines}

The Artemis facility provides a variety of ultrafast, synchronised laser beamlines which can be configured flexibly either to generate XUV or as pump and probe pulses spanning the UV to far infrared. The core of the facility is a $14 \mathrm{~mJ}, 30 \mathrm{fs}$, $1 \mathrm{kHz}$ Ti:Sapphire CPA system operating at $785 \mathrm{~nm}$ (RedDragon from KMLabs). The laser system is carrier-envelope phase (CEP) stabilised, enabling it to provide pulses with precisely defined optical electric fields. The system can achieve $310 \mathrm{mrad}$ rms phase-lock over 1 hour, measured at $13.5 \mathrm{~mJ}$ pulse energy with an integration time of $10 \mathrm{~ms}$ on the f-to-2f interferometer, as shown in Figure 2. The CEP of the oscillator is locked such that every fourth pulse is identical, using a commercial f-to-2f interferometer and electronics (Menlo systems) feeding back to piezo-motors on the oscillator cavity end-mirror to stabilise the path length. Slow CEP drifts occurring during amplification and in the path through the laser system are compensated for with a second f-to- $2 \mathrm{f}$ interferometer, feeding back to piezo-motors on a 
grating in the compressor. Optical stability is provided through cryogenically-cooled amplifier stages where the Ti:Sa crystals are mechanically isolated from the cryo-coolers. The laser system is one of the highest peak- and average-power systems to be CEP-stabilised, and one of the first to implement CEP on a system incorporating multiple amplification stages and grating-based stretcher and compressor. The system runs with a typical pre-pulse on the nanosecond timescale of $0.8 \%$ and with shot-to-shot energy stability of $1 \%$ rms. It can also be run at $3 \mathrm{kHz}$, in which case the maximum pulse energy is in the region of $3.5 \mathrm{~mJ}$.

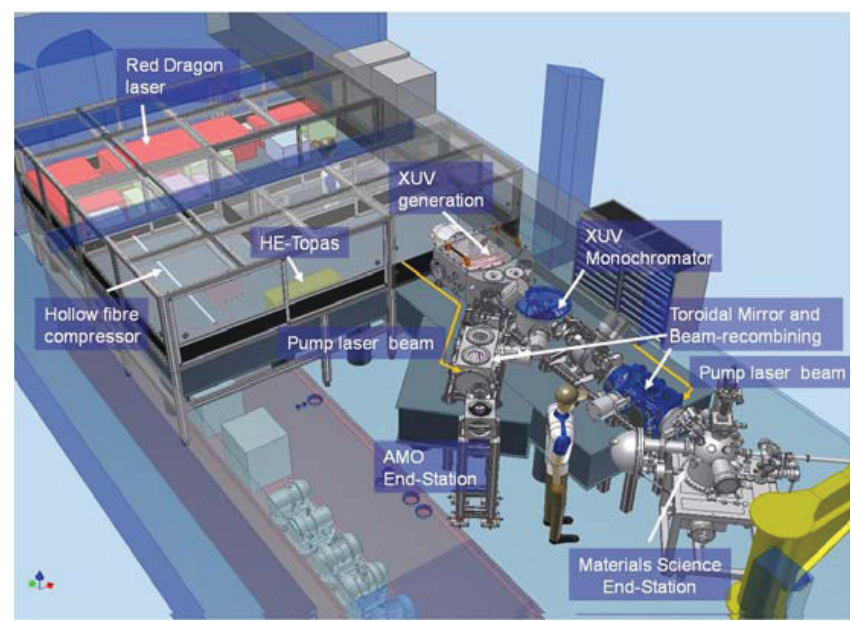

Figure 1. Engineering drawing of the Artemis facility.

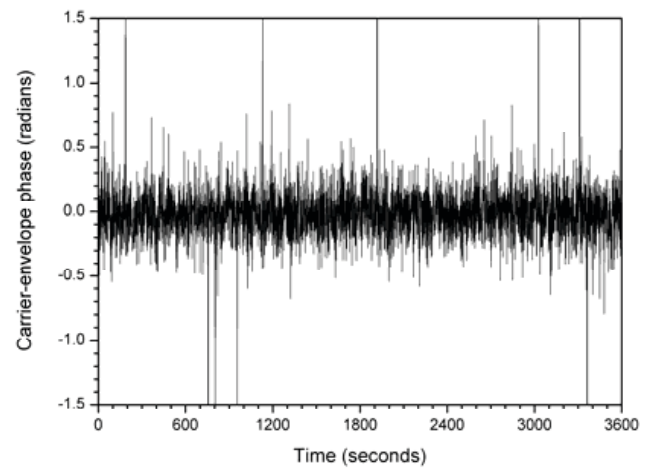

Figure 2. CEP measurement showing an rms value of $310 \mathrm{mrad}$ over a full hour. The laser was operated at $1 \mathrm{kHz}$ with $13.5 \mathrm{~mJ} / \mathrm{pulse}$.

Part of the output energy of the laser can be split and spectrally broadened in a gas-filled hollow fibre and recompressed using chirped mirrors. This technology was successfully implemented in Astra TA1, the precursor facility to Artemis, where $0.3 \mathrm{~mJ}, 10 \mathrm{fs}$ pulses were routinely available. This pulse duration enabled high temporal resolution experiments to be carried out such as, for example, pump and probe imaging of the vibrational wavepacket dynamics ${ }^{8}$ of $\mathrm{HD}^{+}$. We expect that, with the increased bandwidth available from our upgraded chirped mirrors, pulses down to 6 fs with energies in the region of $0.5 \mathrm{~mJ}$ should be achievable on Artemis. This will allow us to provide few-optical cycle pulses with an electric field tailored through CEP-control.

Tuneable pulses spanning the spectral range from $230 \mathrm{~nm}$ to 20 microns are provided by an optical parametric amplifier (HE-Topas from Light Conversion). This is pumped with up to $8 \mathrm{~mJ}$ of the output from the laser system. At $1300 \mathrm{~nm}$, we achieve pulse energies of up to $1 \mathrm{~mJ}(1 \mathrm{~W}$ average power at $1 \mathrm{kHz})$ and measure a pulse duration of $40 \mathrm{fs}$ with a singleshot autocorrelator. This enables focused intensities exceeding $10^{14} \mathrm{Wcm}^{-2}$ to be achieved. The measured tuning curve of the Artemis HE-Topas pumped with 7.7 W from the Red Dragon is shown in Figure 3. Pulse energies in excess of 
$100 \mu \mathrm{J}$ at $250 \mathrm{~nm}$ and $2 \mu \mathrm{J}$ at 20 microns can be obtained. The shot-to-shot energy stability of the output is $3 \% \mathrm{rms}$ with $<2 \%$ rms energy stability from the pump laser.

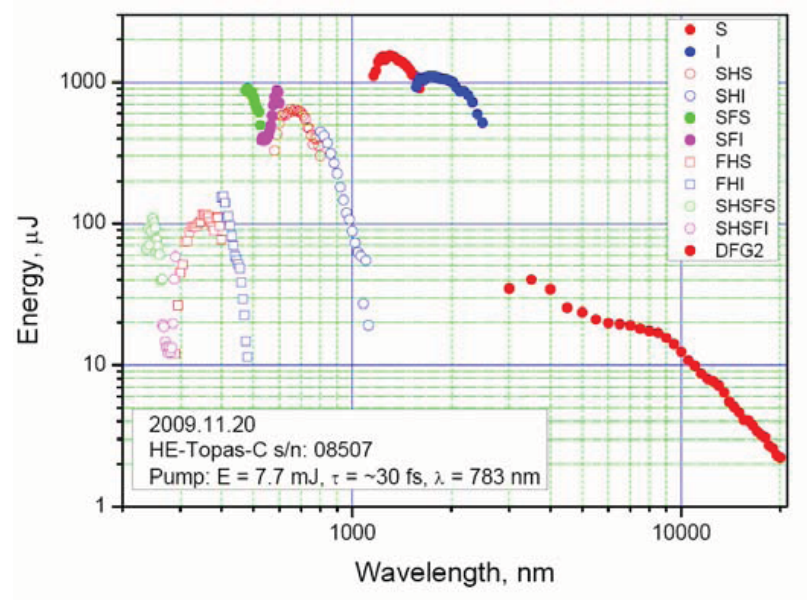

Figure 3. Tuning curve of the HE-Topas pumped with $7.7 \mathrm{~W}$.

\subsection{XUV generation}

XUV radiation in the wavelength range $10-100 \mathrm{~nm}(10-100 \mathrm{eV})$ is produced through high harmonic generation (HHG) in a gas target. The resulting XUV radiation has similar pulse-duration to the drive laser pulse and is synchronised to the drive laser pulse with sub-fs resolution. With conversion efficiencies up to $10^{-6}$ at $30 \mathrm{eV}$, a photon flux of up to $10^{11}$ photons $\mathrm{s}^{-1}$ per harmonic is achievable.

Artemis provides two XUV beamlines - one for monochromatised, narrow bandwidth XUV pulses and one for broadband XUV radiation. There is a single, large, HHG chamber containing two gas jets, one for each XUV beamline. The beamlines are arranged in a Y-geometry as shown in Figure 4, enabling the laser beam to be easily switched from one beamline to the other. For experiments requiring long interaction lengths and minimal gas loads, we use a $\mathrm{kHz}$ pulsed valve with piezo-actuator (Attotech). This has a variety of adaptor nozzles enabling gas path lengths up to $10 \mathrm{~mm}$ and can be used with gas-jet backing pressures up to 5 bar to provide opening times down to $120 \mu$ s. For HHG generation experiments requiring flammable gases or rotational cooling (for molecular gases), we use a continuous flow nozzle, based on a modified Swagelok end-piece with a 100 micron hole drilled through. The HHG chamber is pumped with a 2200 litres/s turbo pump to a base pressure of $10^{-7} \mathrm{mbar}$ with no gas and up to $10^{-3} \mathrm{mbar}$ with gas jet on. With 2 bar of a molecular gas $\left(\mathrm{CO}_{2}\right)$ behind the jet, we measured a rotational temperature of $90 \mathrm{~K}$, sufficiently cold to obtain a significant degree of molecular alignment ${ }^{9}$. The laser beam can be focused into the gas-jet with a lens or curved mirror, with the focal length of typically $20-30 \mathrm{~cm}$ chosen to keep the laser intensity in the region of $10^{14} \mathrm{Wcm}^{-2}$.

Figure 5 shows a calibrated spectrum from argon irradiated with $30 \mathrm{fs}, 800 \mathrm{~nm}$ pulses. This spectrum was measured using the Artemis flat-field spectrometer and represents an average of $\sim 10^{4}$ laser shots. The flat-field spectrometer detects radiation from $20 \mathrm{eV}$ to $80 \mathrm{eV}$ with a resolving power of about 200. The grating is a Hitachi 0266 varied line-spaced spherical grating with a central line density of $1200 \mathrm{~mm}^{-1}$, designed to produce a flat focal plane. The centre of the grating is located $237 \mathrm{~mm}$ from the gas-jet. The detector, consisting of a $40 \mathrm{~mm}$ double stage MCP with a phosphor screen attached and a 14-bit camera, is $235 \mathrm{~mm}$ from the grating. A $2.5 \mathrm{~mm}$ diameter pinhole, located $\sim 200 \mathrm{~mm}$ from the gas-jet enables differential pumping, enabling the pressure in the flat-field spectrometer to be kept below $6 \times 10^{-6} \mathrm{mbar}$ while the gas-jet is running. 


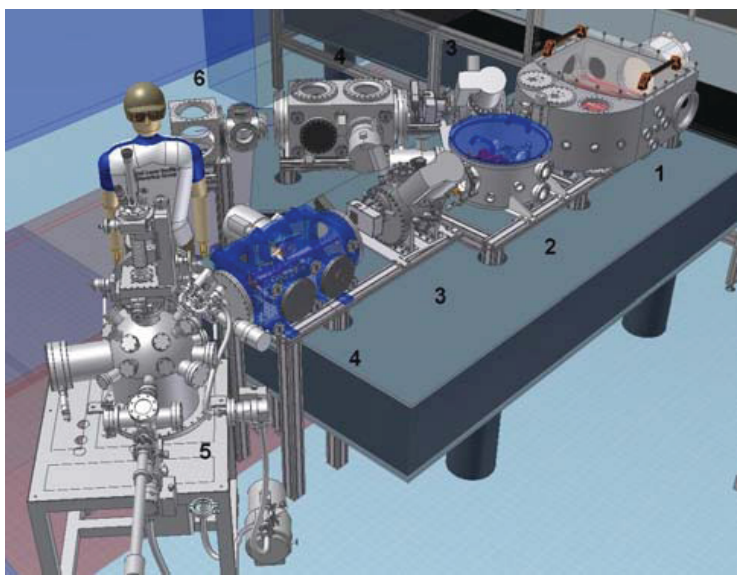

Figure 4. Engineering drawing of the XUV beamlines. Chamber \#1 is the XUV generation chamber, \#2 is the monochromator, \#3 is the flat-field spectrometer (shown in both of its possible locations), \#4 are toroidal mirror chambers, \#5 is the materials science station and \#6 shows the location of the AMO station.

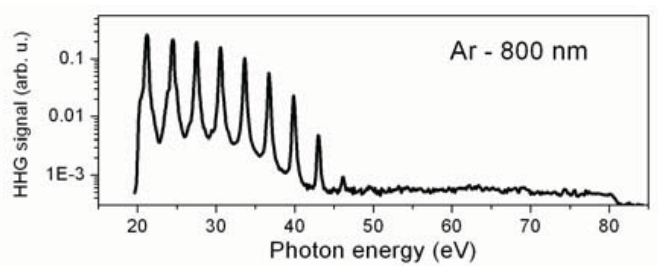

Figure 5. HHG spectrum in Argon, generated with $800 \mathrm{~nm}, 30 \mathrm{fs}$ pulses and detected with the flat-field spectrometer.

\subsection{Narrow bandwidth XUV beamline}

For experiments requiring wavelength and bandwidth selection, Artemis provides a narrow-bandwidth, tuneable XUV beamline. In this beamline the harmonics pass through a state of the art XUV monochromator, which enables a single harmonic, or narrower bandwidth, to be selected from the spectrum, while maintaining the femtosecond pulse-duration.

The XUV monochromator has been designed and built in collaboration with the LUXOR Lab, Italian National Research Council. The monochromator consist of a toroidal mirror, which collimates the XUV radiation and directs it on to a plane diffraction grating. The diffracted light is refocused by a second toroidal mirror on to the exit slit of the monochromator, as shown in Figure 6. The grating is used in conical illumination (with the direction of light propagation nearly parallel to the plane of the rulings) to limit the time spread of the pulses and maintain the required resolving power and high efficiency ${ }^{10,11,12}$.

The monochromator is equipped with four interchangeable diffraction gratings to cover two spectral ranges with either higher resolving power or shorter pulse duration, as shown in Table 1 . The resolution is limited to $\lambda / \Delta \lambda \sim 100$ in order to keep the XUV pulse duration below 60 fs. The single grating design results in a measured peak XUV transmission of $30 \%$. The monochromator is designed to image a $20 \mu \mathrm{m}$ XUV source on to the exit slit. As the focused intensity should be kept to $\sim 10^{14} \mathrm{Wcm}^{-2}$ for harmonic generation, this limits the amount of laser energy that can be usefully used to generate harmonics to $<1 \mathrm{~mJ}$.

The XUV flux after the monochromator is measured with a calibrated XUV channeltron which can be inserted into and removed from the XUV beamline without breaking vacuum. We measure a flux of up to $1.6 \times 10^{10}$ photons $/ \mathrm{second}$ at $32.5 \mathrm{eV}$ photon energy (harmonic 21) after the monochromator. This corresponds to an XUV energy of $80 \mathrm{pJ} / \mathrm{pulse}$, with a pulse repetition rate of $1 \mathrm{kHz}$ and a monochromator bandwidth of $900 \mathrm{meV}$. When the monochromator is used 
with maximum resolution we obtain a photon flux of $9 \times 10^{9}$ photons/second at $32.5 \mathrm{eV}$ photon energy with a bandwidth of $390 \mathrm{meV}$. Figure 7 shows the scanned HHG spectrum obtained with the XUV monochromator and grating \#3.

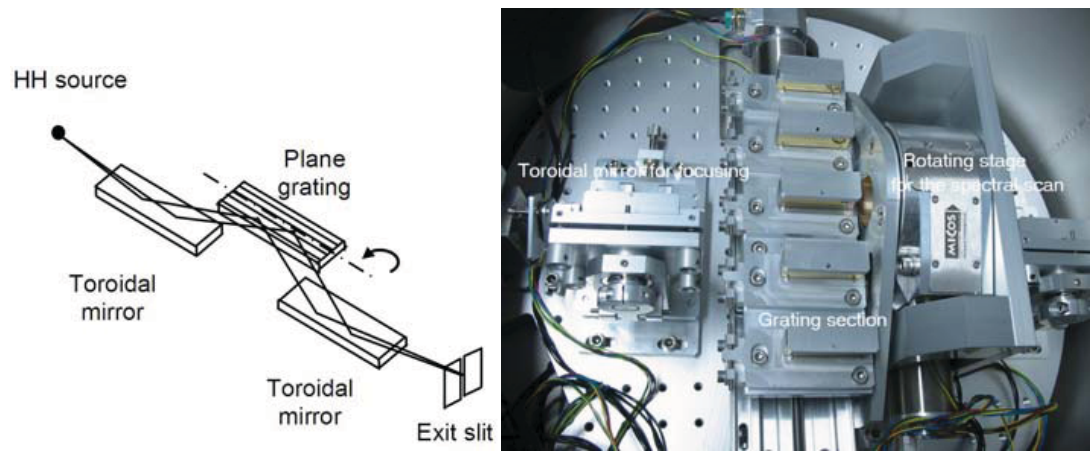

Figure 6. Schematic of the monochromator (left) and internal view of the instrument (right), showing the two toroidal mirrors and four interchangeable gratings.

\begin{tabular}{|c|c|c|c|}
\hline Grating & Tuning range & $\begin{array}{c}\text { Shortest XUV pulse } \\
\text { duration }\end{array}$ & Resolution \\
\hline Grating 1 & $20-40 \mathrm{eV}(62-31 \mathrm{~nm})$ & $10 \mathrm{fs}$ & $\lambda / \Delta \lambda=26 @ 46 \mathrm{~nm}(27 \mathrm{eV})$ \\
\hline Grating 2 & $100-35 \mathrm{eV}(12-35 \mathrm{~nm})$ & $10 \mathrm{fs}$ & $\lambda / \Delta \lambda=23 @ 20 \mathrm{~nm}(62 \mathrm{eV})$ \\
\hline Grating 3 & $20-40 \mathrm{eV}(62-31 \mathrm{~nm})$ & $56 \mathrm{fs}$ & $\lambda / \Delta \lambda=100 @ 46 \mathrm{~nm}(27 \mathrm{eV})$ \\
\hline Grating 4 & $100-35 \mathrm{eV}(12-35 \mathrm{~nm})$ & $40 \mathrm{fs}$ & $\lambda / \Delta \lambda=70 @ 20 \mathrm{~nm}(62 \mathrm{eV})$ \\
\hline
\end{tabular}

Table 1. Design performance of the Artemis XUV monochromator for each of the four gratings.

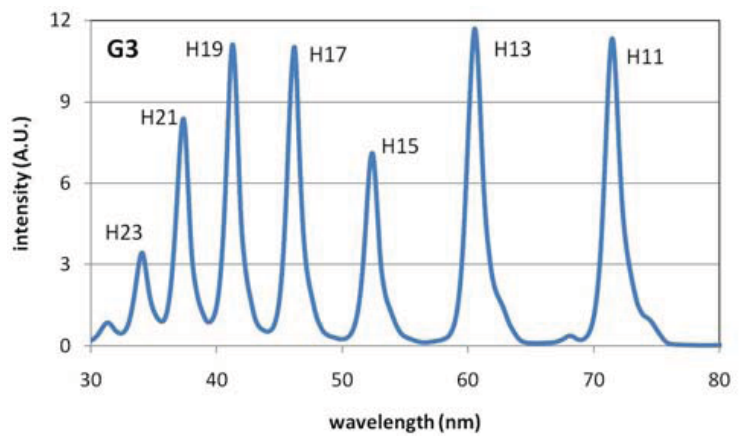

Figure 7. HHG spectrum generated with $1 \mathrm{~mJ}$ of $800 \mathrm{~nm}$ radiation in argon, obtained using the monochromator and grating 3.

A toroidal mirror with gold coating on a Zerodur substrate refocuses the XUV inside the end-station. The focal length of the toroidal mirror is $47 \mathrm{~cm}$ and it is set up in a $4 \mathrm{f}$ geometry, with $94 \mathrm{~cm}$ from the monochromator exit slit to the centre of the mirror and $94 \mathrm{~cm}$ from the centre of the mirror to the focus inside the end-station. The $10 \mathrm{~mm} \times 143 \mathrm{~mm}$ toroidal mirror is used at a $4^{\circ}$ grazing angle ( $86^{\circ}$ angle of incidence) and is polished to $3 \mathrm{~nm}$ roughness, giving a reflectivity of $80 \%$ for wavelengths longer than $8 \mathrm{~nm}$. The toroidal mirror position is optimised using IR pulses transmitted by setting the monochromator to zero-order and fine-tuning the five motorised axes of motion of the mirror. In this way, we have achieved IR focal spot sizes of $130 \times 90 \mu \mathrm{m}$. As there is one-to-one imaging through the monochromator and toroidal mirror system, the XUV spot size will be at best the same as the XUV source size, estimated to be $\sim 20 \mu$ m, much smaller than the IR focal spot. 
The XUV pulses can be recombined with auxillary laser pulses for pump-probe experiments in several geometries. The auxillary laser pulse propagates parallel to the XUV beamline down an external, enclosed path, is focused and then recombined with the XUV pulse - either by reflection off a mirror with a central hole that the XUV passes through or by reflection in a non co-linear geometry. The end-stations used for experiments on Artemis typically require vacuum levels of $10^{-8}$ to $10^{-10}$ mbar to operate. To achieve these vacuum levels in a beamline where the HHG chamber is at $10^{-3} \mathrm{mbar}$ requires several stages of differential pumping. The monochromator is separated from the HHG chamber by a $\sim 2 \mathrm{~mm}$ pinhole and typically reaches the $10^{-6} \mathrm{mbar}$ level when the gas-jet is on (its base pressure is $10^{-7} \mathrm{mbar}$ ). The exit slit of the monochromator provides differential pumping to the toroidal mirror chamber $\left(10^{-7} \mathrm{mbar}\right)$. A pair of pinholes surrounding a small cube chamber provide an additional stage of differential pumping and allow vacuum levels down to $8 \times 10^{-10}$ mbar to be achieved in the interaction station while the gas-jet is running. As there are typically no optics between the toroidal mirror and the end-station, the best focus of the toroidal mirror can only be obtained in one position. This means that the end-station needs to be carefully aligned and centred around the XUV focal spot.

\subsection{XUV pulse duration measurement}

The duration of XUV pulses from the monochromator has been measured and showed that the monochromatised XUV pulses had a sub-30 fs pulse-duration for a $28 \mathrm{fs}$ IR drive pulse. The experimental set up is shown in Figure 8 and is typical of the time-resolved experiments planned for the monochromatised XUV beamline. The $28 \mathrm{fs}, 780 \mathrm{~nm}$ laser beam is split into two by a beam-splitter (BS). One laser beam is focused into the argon gas jet in the HHG chamber generating the XUV beam containing significant photon flux from harmonics H11 to H29. This beam passes through the XUV monochromator and is focused by a toroidal mirror (TM3) into a second gas-jet (J2) in the ion time-of-flight spectrometer (Ion TOF), which has previously been employed to study strong-field ionization effects in argon ${ }^{13}$. The second laser beam passes through a variable delay stage, propagates parallel to the XUV beamline, is focused by a lens and recombined with the XUV beam into the ion ToF spectrometer. The two beams are recombined at an annular mirror which reflects the IR laser beam and has a hole in the centre, through which the XUV beam passes.

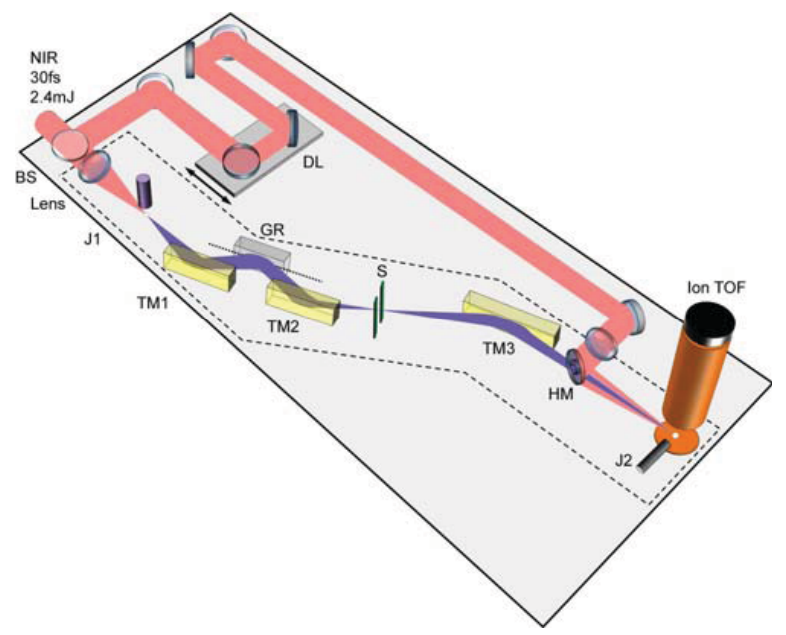

Figure 8. Experimental set-up for measuring the duration of monochromatised XUV pulses. BS is a beamsplitter, J1 and J2 are gas jets, DL is the delay line, $\mathrm{S}$ is a slit, TM1, TM2 and TM3 are toroidal mirrors, HM is a holey mirror, Ion ToF is an ion time-of-flight mass spectrometer. The dashed line indicates the vacuum chamber presented in Figure 4.

All pump-probe measurements require that the temporal overlap be known, defined by first observing the spatial overlap of the pump and probe beams in the IR. The monochromator is tuned to the zero-order, propagating the IR co-linearly with the harmonics through the XUV beamline, allowing the focal spots in the ion spectrometer to be observed with a CCD over the full range of the delay line $(\sim 1.667 \mathrm{~ns})$. The temporal overlap is then found by scanning the zero-order (XUV+IR) pump and IR probe and observing interference fringes on the exit of the end-station.

The temporal overlap is then observed in the ion spectrometer by measuring the single ionization yield of krypton, requiring an IR intensity of $\sim 10^{13} \mathrm{Wcm}^{-2}$ or a photon energy of more than approximately $14 \mathrm{eV}$ depending on the 
ionization regime. The monochromator is operated in the zero-order configuration hence the IR components in each arm form an interferometer with $\sim 6 \mathrm{~m}$ arms, and as the delay line is scanned, temporal fringes are clearly apparent in the measured $\mathrm{Kr}^{+}$yield presented in Figure 9. Fine structure with $1 / 2$ laser-cycle, (1.3 fs) is clearly resolved and shows that the interferometer was stable to better than $1 / 4$ cycle $(0.6 \mathrm{fs})$ over this measurement. These fringes were still visible after 12 hours, showing that the two foci remained spatially and temporally overlapped over this time. This gives us confidence that challenging multi-pulse femtosecond pump-probe experiments using the XUV beamlines can be attempted.

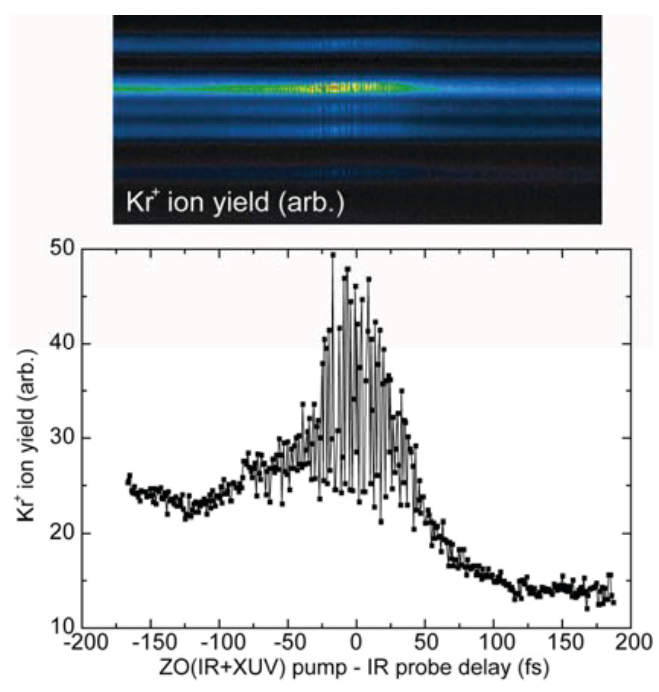

Figure 9. Zero-order (IR + XUV) pump, IR probe measurement, showing interference fringes between the two IR pulses. The asymmetry in the $\mathrm{Kr}+$ yield is a consequence of the XUV also transmitted in the zero-order.

Were the pump and probe pulses employed in Figure 9 purely IR, a temporally symmetric distribution would result; the asymmetry visible at large positive and negative delay is a result of the harmonic photons transmitted by the monochromator. Therefore, by rotating the grating (GR in Figure 8) to transmit a single harmonic we observe single XUV photon absorption followed by IR strong field ionization. The energy level structure of krypton is such that an XUV pump photon can be absorbed to generate a highly excited state of $\mathrm{Kr}^{+}$through the emission of a $4 \mathrm{~s}$ or $4 \mathrm{p}$ electron, then the strong-field IR probe will easily ionize to $\mathrm{Kr}^{2+}$, as presented in Figure 10. The temporal "step" indicates that if the pump and probe are reversed in time, no energetically favourable route to $\mathrm{Kr}^{2+}$ exists. An XUV photon (H23, $35.7 \mathrm{eV}$ ) generates the $\mathrm{Kr}^{2+}$ population on the condition that the strong field pulse is also present, hence the shape of the step function is a measure of the temporal cross-correlation. The convolution of a 28 fs Gaussian IR pulse with the cumulative distribution function of a Gaussian of a variable duration is overlaid on the experimental data, indicating the XUV pulse must have a duration of 20-30 fs. These measurements were made with the monochromator slit $160 \mu \mathrm{m}$ wide and the XUV bandwidth $\lambda / \Delta \lambda \sim 20$. The XUV pulse duration increases to $\sim 60 \mathrm{fs}$ when the XUV bandwidth is reduced to $\lambda / \Delta \lambda \sim 30$ by closing the monochromator slit to $110 \mu \mathrm{m}$ width.

The temporal and spatial resolution and stability of the Artemis beamlines is the result of good design and engineering. This includes air conditioning with laminar flow through a perforated ceiling from an air-conditioning unit outside building and water-to-water laser chillers with closed loop heat exchanger also outside the building. The beamline is built on a solid, monolithic optical bench, contiguous with the laser system, with optics breadboards inside vacuum chambers isolated from chamber walls and turbo-pumps but rigidly connected to the bench. As much equipment as possible, including scroll pumps, cryo-compressors, and laser chillers are placed in a concrete trench under part of the lab floor. 


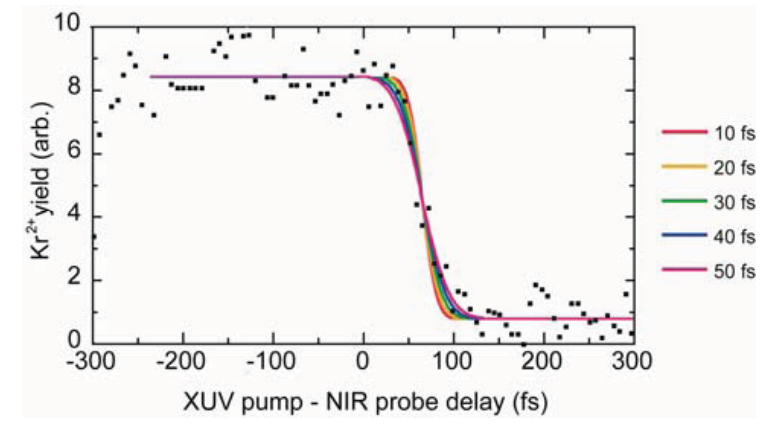

Figure 10. Yield of $\mathrm{Kr} 2+$ with harmonic 23 pump and $800 \mathrm{~nm}$ probe as a function of the delay between the two pulses.

\subsection{Broadband XUV beamline for attosecond science}

The second XUV beamline on Artemis is a broadband beamline, without a monochromator. This beamline has been designed to be compatible for attosecond science, generating the attosecond XUV pulses using a few-cycle CEPstabilised pulse. The XUV generation region in the gas-jet is imaged onto the interaction region in the end-station by an identical toroidal mirror set-up to the monochromatised beamline. Wavelength selection on this beamline is provided through appropriate filtering. A key design consideration for this beamline is the rejection of the drive laser pulse, which co-propagates with the harmonics. One option provided in this beamline will be a pair of XUV-IR beamsplitters, where the IR beam is transmitted at Brewster's angle through an optic highly reflective in the XUV. Silica has been shown to be a suitable material for wavelengths above $10 \mathrm{~nm}$, attenuating the IR by a factor of $10^{-4}$ per surface ${ }^{14}$, while reflecting $30 \mathrm{~nm}$ XUV pulses with a reflectivity of 0.56 . This design also allows a probe pulse of different wavelength to be recombined by injection through the back of the second beamsplitter and permits the position of the XUV focus to be moved inside the target chamber without affecting the focusing.

\section{FEMTOSECOND TIME-RESOLVED SCIENCE END-STATIONS}

\subsection{Materials Science end-station}

The Artemis Materials Science end-station (Figure 11) is designed for time- and angle-resolved photoemission spectroscopy. The end-station is a UHV chamber, which pumps to $<2 \times 10^{-10} \mathrm{mbar}$, equipped with a hemispherical electron analyser (SPECS Phoibos 100) and two-dimensional detector for energy- and angle-resolved measurements with an energy resolution of $\sim 5 \mathrm{meV}$ and an angular resolution of $<1^{\circ}$. A liquid-helium-cooled, five-axis manipulator enables the sample to be cooled to $14 \mathrm{~K}$ or resistively heated to $1000 \mathrm{~K}$. The sample can be cooled from room temperature down to $14 \mathrm{~K}$ in $<30$ minutes.

The station consists of a spherical main chamber constructed from mu-metal, to reduce the effect of stray magnetic fields. A separate load-lock chamber contains a magnetic transfer arm which allows the sample to be easily transferred to the sample manipulator, with a sample turn-around time of $\sim 1$ hour, greatly reducing the down-time associated with vacuum pumping and bake-out. The chamber includes a low-energy electron diffraction (LEED) apparatus to characterise sample surfaces. A helium discharge lamp emitting at $21.2 \mathrm{eV}$ enables off-line alignment of the analyser and calibration of the energy resolution. In the future, we hope to include provision for a wide-range of sample preparation techniques, such as an ion gun for sample sputtering and evaporators for thin film growth.

The station has been integrated with the narrow bandwidth XUV beamline in a commissioning experiment to study femtosecond time-resolved dynamics of Mott insulators. The first angle-resolved photoelectron measurement is shown in (Figure 12). Radiation with a wavelength of $37 \mathrm{~nm}$ (harmonic 21) was focused onto a $\mathrm{TaS}_{2}$ crystal $^{15}$ at $14 \mathrm{~K}$. In this measurement, there were approximately $10^{7}$ XUV photons per pulse in a diameter of $\sim 300$ microns. The photon flux was adjusted in order to avoid space charge effects while maintaining a reasonable acquisition time. The current overall resolution is $250 \mathrm{meV}$ at the Fermi edge, which may be associated with the XUV bandwidth. 


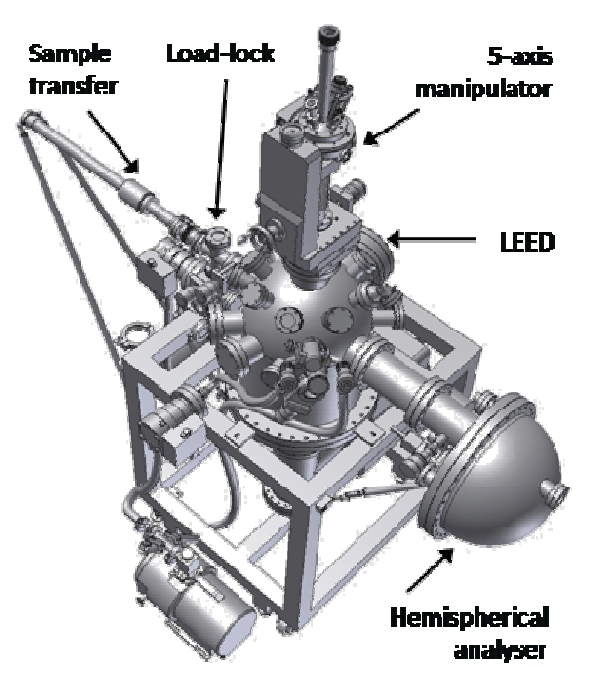

Figure 11. The Artemis Materials Science station, which includes a hemispherical electron analyser for time- and angle-resolved photoelectron spectroscopy.

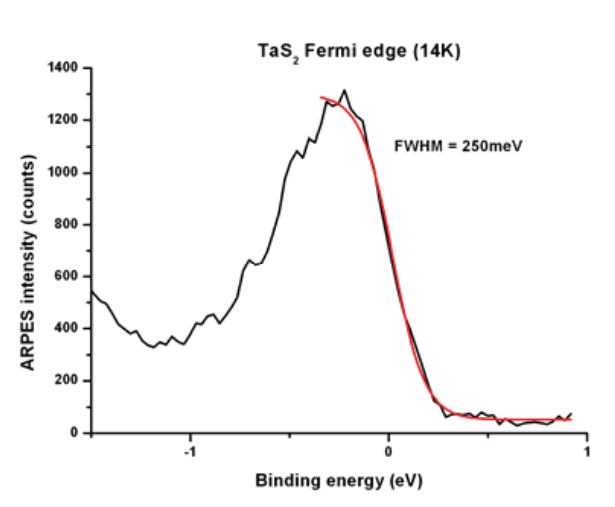

Figure 12. Measurement of the $\mathrm{TaS}_{2}$ Fermi edge at $33.5 \mathrm{eV}$ (photon energy $37 \mathrm{~nm}$ ).

The Artemis Materials Science End-Station is well suited for angle- and time-resolved investigations of photoelectron emission for science such as: coherent control and Fermi surface dynamics in complex oxides; non-adiabatic melting of charge order and Mott-gap dynamics; ultrafast core-level electron photoemission. The short wavelength of the XUV pulse allows access to a larger region of the Brillouin zone than would be possible in a laser system using lower harmonics, while maintaining femtosecond time resolution which standard synchrotron radiation cannot provide.

\subsection{Atomic and Molecular Physics and Chemistry}

The atomic and molecular physics end-station has been designed for experiments on gas targets and is currently under construction. This interaction station will permit experiments such as studies of the dynamics of aligned molecules, control of electron recollisions, time-resolved photoelectron imaging of excited state molecular processes, and Coulomb explosion imaging of molecular wavepackets. Future development of the molecular beam source will allow studies on clusters and molecules of biological interest.

The interaction station consists of two coupled chambers. The lower chamber contains a molecular beam source for producing molecules rotationally cooled to $<10 \mathrm{~K}$ and is pumped by two turbo pumps with a combined pumping speed of $\sim 6000$ litres $\mathrm{s}^{-1}$. A skimmer with a $0.8 \mathrm{~mm}$ diameter nozzle provides differential pumping. An upper section contains a velocity-map imaging (VMI) detector for ions and electrons. In the interaction region of the VMI, the cool, collimated 
beam of target gas intercepts the laser or XUV pulse. The molecule or cluster is ionised by the laser and ions or electrons produced are accelerated by a series of electrodes onto a $75 \mathrm{~mm}$ diameter two-stage imaging microchannel plate. The flight path is enclosed in a double layer of mu-metal to shield the charged particles from stray magnetic fields. The electrodes are carefully designed so that ions (or electrons) with the same initial velocity but created in different parts of the interaction region arrive at the same point on the detector. By applying an inversion algorithm to the resulting image, the original 3D velocity distribution of the particles can be recovered. The VMI detector also has the advantage that particles ejected at all emission angles can be collected, meaning that the full angular distribution can be measured without the need to rotate either the detector or the laser polarisation. Voltages of up to $15 \mathrm{kV}$ can be applied to the electrodes, with the goal of allowing electrons with energies up to $10 \mathrm{~s}$ to $100 \mathrm{~s}$ of eV to be imaged. The spectrometer may also be configured for time-of-flight detection of ions or electrons.

\section{NEW SCIENCE ON ARTEMIS}

\subsection{Photodissocation of $\mathrm{D}_{3}^{+}$}

One of the most significant mechanisms by which molecules are dissociated in an intense laser field is that of bond softening. This process usually occurs for the molecular cation following ionization of the neutral molecule and the strength of the coupling between electronic states by the electric field of the laser is highly dependent on the internal energy. As the cation is typically formed in a range of vibrational states, contributions from each of these initial states can be hard to disentangle in experiments.

To perform experiments on molecular cations in a well-defined state, a pioneering ion trapping experiment was performed using the Astra TA1 laser facility ${ }^{16}$, the precursor to Artemis. In this study, $\mathrm{HD}^{+}$ions were confined in a linear, electrostatic ion trap for periods beyond 0.3 seconds. This allowed the ions to cool down into their ground vibrational state before a $40 \mathrm{fs}$ laser pulse induced dissociation. This showed that more than the minimum number of photons could be absorbed in the dissociation process, something which had not been observed in previous studies.

A consequence of creating these "cold" molecules is that they are more stable in the laser field, reducing the signal rate considerably. The order of magnitude increase in pulse energy available at the new Artemis facility has made these experiments more accessible and has opened up the study of other highly stable molecules. One such molecule is the most fundamental triatomic molecule $\mathrm{H}_{3}{ }^{+}$. Using 30 fs pulses from Artemis, the isotopic variant $\mathrm{D}_{3}{ }^{+}$was dissociated in a strong $800 \mathrm{~nm}$ field for the first time. The $\mathrm{D}_{3}{ }^{+}$target was prepared in a novel linear electrostatic storage device ${ }^{17}$ (Figure 13).

Using a 'Z-scan' technique where the laser focus is translated along the laser propagation direction, a time-of-flight spectrum of the neutral dissociation fragments was obtained at each z position (Figure 14). The data was obtained for ions trapped for less than $500 \mu \mathrm{s}$, corresponding to vibrationally 'hot' ions. When the $\mathrm{D}_{3}{ }^{+}$target was positioned at the focus of the laser, a noticeable dip is seen in the dissociation signal. This may initially seem surprising, as the highest intensity is experienced at this point. However, the small overlap volume between the laser focus and the ion beam reduces the magnitude of the signal. When the focal position is at $\mathrm{z}= \pm 6 \mathrm{~mm}$, there is a noticeable peak in the dissociation yield. Although the intensity has decreased at this point (compared to smaller $z$ values), the volume has significantly increased. At $\mathrm{z}= \pm 9 \mathrm{~mm}$, even though the overlap volume increases further, the signal begins to drop due to a decrease in intensity. By modelling the overlap of the focussed laser with the ion beam, the trend of the data has been found to be consistent with a multi-photon process of 2 or 3 photons.

The dissociation signal was also studied as a function of trapping time. For interactions occurring during the first millisecond of trapping, the dissociation signal was found to remain steady and even increase slightly. This was followed by a drop of two orders of magnitude between 1 and $10 \mathrm{~ms}$ with no observed signal beyond this.

These results indicate that even with the high pulse energies available at Artemis, it is only very highly excited states close to the dissociation limit that are contributing to the dissociation. Once these states have decayed in the trap (around $10 \mathrm{~ms}$ ), the molecule is found to be very stable. This can be attributed to the large energy separation between the ground electronic state in its equilibrium configuration (an equililateral triangle) and any excited states ( $>17.5 \mathrm{eV})$. In a highly excited state, an elongated geometry makes transitions between $1^{1} \mathrm{~A}^{\prime}$ and $2^{1} \mathrm{~A}^{\prime}$ states possible via 2 or 3 photon absorption $^{18}$. 


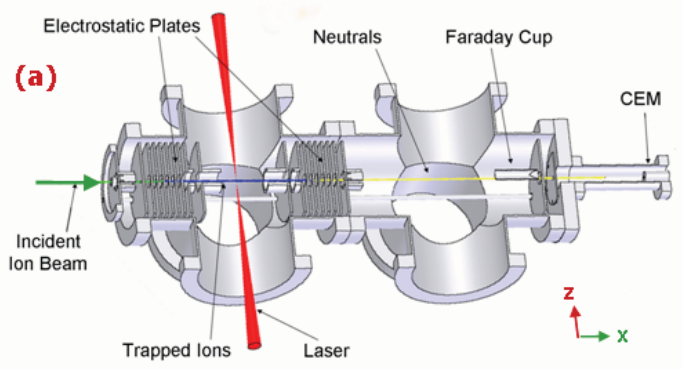

(b)

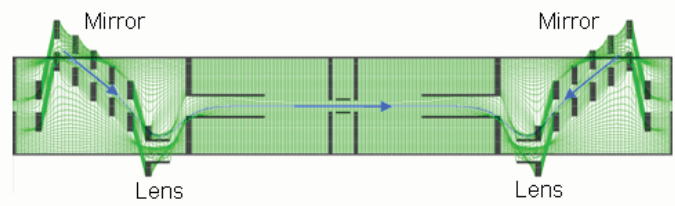

Figure 13. Linear electrostatic storage device used to prepare $\mathrm{D}_{3}{ }^{+}$target for intense-field studies. (a) cut-away of the device (b) potential surface experienced by a positive ion in the trapping region. Low-energy $(1 \mathrm{keV})$ ions were confined in the electrostatic cavity, where electrostatic plates provide axial confinement (mirrors) and radial confinement (lens). Neutrals from the laser interaction will escape the trapping region and be detected at the channel electron multiplier (CEM).

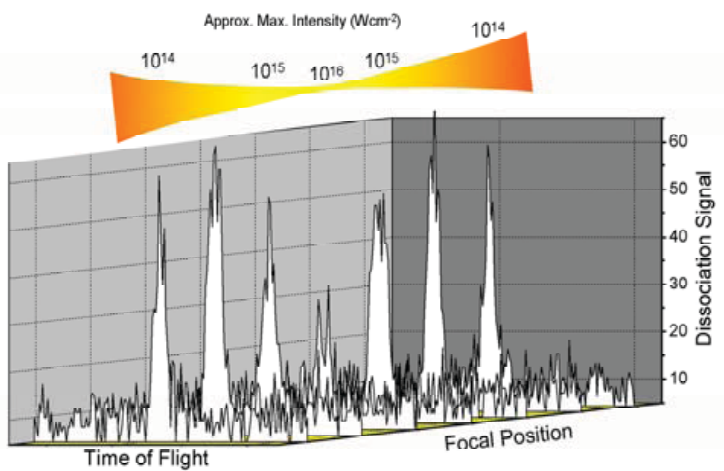

Figure 14. Z-scan of $\mathrm{D}_{3}{ }^{+}$dissociation in intense fs pulses. The yield from neutral fragments is found as a function of the laser focus zposition. The focal position was varied from $z=-9 \mathrm{~mm}$ to $\mathrm{z}=+0 \mathrm{~mm}$ in steps of $3 \mathrm{~mm}$.

\subsection{Probing molecular structure and dynamics with mid-IR HHG in aligned molecules.}

The mid-IR radiation available from the optical parametric amplifier is especially suitable for the generation of high harmonics in molecular systems with low ionization potential. Figure 15 shows two harmonic spectra from acetylene (IP $=11.4 \mathrm{eV}$ ). The spectrum in the upper panel was obtained using the $10 \mathrm{~Hz}$ Astra TA1 facility operating at $800 \mathrm{~nm}^{19,20}$. With pulses of $10 \mathrm{fs}$ the highest photon energy in the spectrum could not be extended beyond $46 \mathrm{eV}$ due to saturation of the ionization. The lower panel shows the spectrum obtained in Artemis with $40 \mathrm{fs}, 1300 \mathrm{~nm}$ pulses. In this case, even at an intensity below the saturation limit, the maximum harmonic energy reaches $60 \mathrm{eV}$.

This remarkable increase in photon energy facilitated by the mid-IR field allowed the observation for the first time of structural interference signatures in the harmonic spectra of molecules with a low ionization potential like acetylene $\left(\mathrm{C}_{2} \mathrm{H}_{2}\right)$ and nitrous oxide $\left(\mathrm{N}_{2} \mathrm{O}\right)^{9}$. The structural interference signatures are observed as a suppression of harmonic signal in the spectra recorded when the molecules are aligned by an $800 \mathrm{~nm}$ laser pulse (Figure 16). 

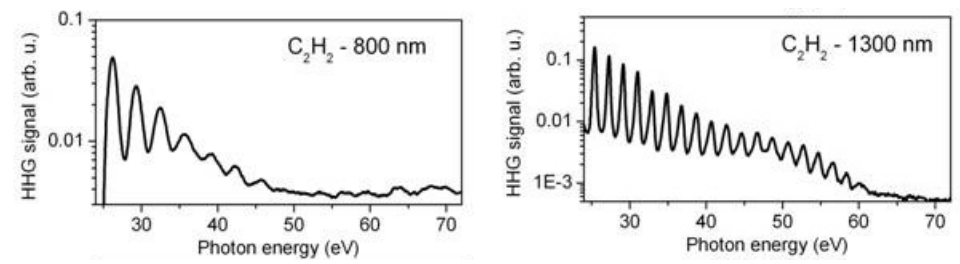

Figure 15. Measured XUV HHG spectra in acetylene ( $\mathrm{IP}=11.4 \mathrm{eV}$ ) using $10 \mathrm{fs}, 800 \mathrm{~nm}$ pulses at $1.8 \times 10^{14} \mathrm{~W} / \mathrm{cm}^{2}$ (left) and $40 \mathrm{fs}$, $1300 \mathrm{~nm}$ pulses at $1.0 \times 10^{14} \mathrm{~W} / \mathrm{cm}^{2}$ (right). The spectra are not normalized.

The use of a $1300 \mathrm{~nm}$ fields for high harmonic generation also has an advantage for molecules with a high ionization potential like $\mathrm{CO}_{2}(\mathrm{IP}=13.8 \mathrm{eV})$. As the mid-IR field requires much less laser intensity than a $800 \mathrm{~nm}$ field to reach the same photon energies, a wider range of intensities is available to perform intensity-dependence studies of harmonic generation.

One such study, performed in aligned samples of $\mathrm{CO}_{2}$ has revealed the interplay between the interference that arises from the static two-centre structure of the molecules and a dynamic interference between different ionization channels ${ }^{21}$. These two effects were observed separately in previous experiments and were long considered as differing explanations of the same phenomenon. The new measurements carried out in Artemis have reconciled the two opposed views. This new science shows Artemis' capability in the new field of real-time observation of molecular orbital dynamics (electron hole motion) on an attosecond timescale with sub-nm spatial and femtosecond temporal resolution.
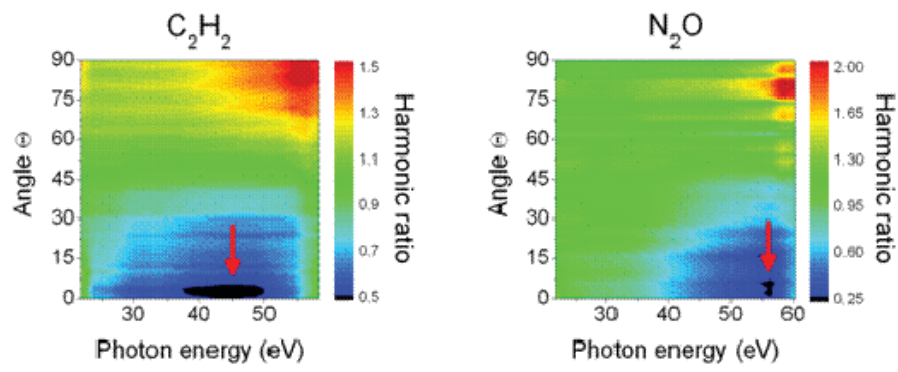

Figure 16. Ratio of harmonic signal between aligned and unaligned samples of $\mathrm{C}_{2} \mathrm{H}_{2}$, and $\mathrm{N}_{2} \mathrm{O}$ as a function of photon energy (harmonic order) and polarization angle $\Theta$ of the aligning pulse for a driving laser pulse at $1300 \mathrm{~nm}$. The red arrows mark the position of the interference minima.

\subsection{Enhancement of HHG by a sum of multiple unrelated frequencies.}

The availability of multiple laser wavelengths from the Artemis facility was used to investigate the enhancement of HHG photon flux by a sum of multiple unrelated frequencies ${ }^{22}$. The fundamental $780 \mathrm{~nm}$ laser beam was mixed with the $1300 \mathrm{~nm}$ beam from HE-Topas in a gas-jet of argon with different relative intensities. The addition of a small portion of $780 \mathrm{~nm}$ radiation to the $1300 \mathrm{~nm}$ field, with parallel polarizations, was found to compensate the decrease of harmonic signal observed in harmonic generation with mid-IR fields while maintaining, or even increasing, the higher photon energy achieved by the long wavelength field. An enhancement of more than two orders of magnitude was observed in the intensity of the harmonics of the two-colour field as compared to $1300 \mathrm{~nm}$ alone, and non-integer order harmonics were observed (Figure 17). 

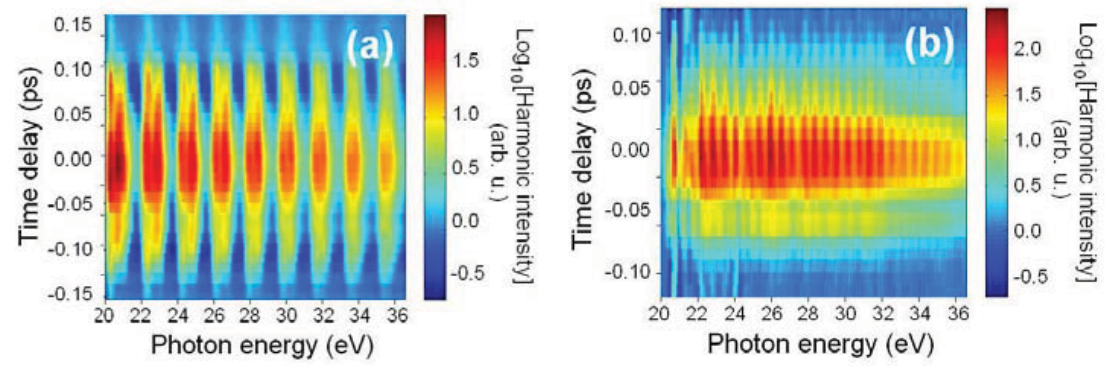

Figure 17. Measured harmonic spectra of argon as a function of delay between $\lambda_{1}=1300 \mathrm{~nm}$ and $\lambda_{2}=780 \mathrm{~nm}$ pulses, with parallel polarisation (normalised to the harmonic intensity of the $1300 \mathrm{~nm}$ field alone). (a) $\mathrm{I}_{1}=1.5 \times 10^{14} \mathrm{~W} / \mathrm{cm}^{2} ; \mathrm{I}_{2}=0.2 \times 10^{14} \mathrm{~W} / \mathrm{cm}^{2}$. (b) $\mathrm{I}_{1}=$ $0.5 \times 10^{14} \mathrm{~W} / \mathrm{cm}^{2} ; \mathrm{I}_{2}=0.5 \times 10^{14} \mathrm{~W} / \mathrm{cm}^{2}$.

\section{CONCLUSIONS}

The Artemis facility for ultrafast XUV science at the Central Laser Facility in the UK aims to capitalise on recent advances in ultrafast laser technology by bringing them together in combination to provide a flexible XUV source for a wide variety of experiments. Artemis is constructed around a high-average power carrier-envelope phase-stabilised system, which is used to generate tuneable pulses across a wavelength range spanning the ultraviolet to the far infrared, few-cycle pulses at $800 \mathrm{~nm}$ and short pulses of XUV radiation produced through high harmonic generation. The XUV pulses can be delivered to interaction stations for materials science and atomic and molecular physics and chemistry through two vacuum beamlines for broadband XUV or narrow-band tuneable XUV pulses. The novel XUV monochromator enables a single harmonic to be selected from the spectrum. Measurements of the XUV pulse duration using an XUV-pump IR-probe technique demonstrate that the monochromator preserves the duration of the XUV pulses to less than $30 \mathrm{fs}$. The materials science station, which contains a hemispherical electron analyser and five-axis manipulator cooled to $14 \mathrm{~K}$, is optimised for photoemission experiments with the XUV. The end-station for atomic and molecular physics and chemistry includes a velocity-map imaging detector and molecular beam source for experiments on the time-resolved photoelectron imaging, dynamics of aligned molecules, control of electron recollisions and Coulomb explosion imaging of molecular wavepackets.

A key goal of the Artemis facility is to make XUV pulses available for applications, by combining expertise and technologies from the femtosecond, optical domain and synchrotron science. The ultrafast XUV science carried out on Artemis is a precursor to the science anticipated on free electron lasers. As such, Artemis adds a significant and highly strategic capability to the UK scientific community. The facility is now fully operational and open to UK and European users for twenty weeks per year. Some of the key new scientific results obtained on the facility include; the first observation of $\mathrm{D}_{3}{ }^{+}$photodissociation in strong fields; extension of HHG imaging spectroscopy to the mid-infrared; enhancement of the conversion efficiency in HHG by combining two laser fields with non-harmonically related wavelengths. Future upgrades will aim to build Artemis into an open-access facility for attosecond science.

\section{ACKNOWLEDGEMENTS}

We thank CLF engineering staff, particularly Steve Hook, Simon Spurdle, Brian Landowski and Dan Wolff, for their work to build the facility and support experiments. This work was financially supported by the STFC Facility Development programme, STFC facility access grants and Laserlab Europe.

\section{REFERENCES}

[1] Krausz F. and Ivanov M., “Attosecond Physics”, Rev. Mod. Phys. 81, 163 (2009).

[2] Kling M.F. and Vrakking M.J.J., “Attosecond electron dynamics”, Ann. Rev. Phys. Chem. 59463 (2008).

[3] Baltuska A., Udem T., Uiberacker M., Hentschel M., Goulielmakis E., Gohle C., Holzwarth R., Yakoviev V. S. , Scrinzi A., Hansch T. W., Krausz F., “Attosecond control of electronic processes by intense light fields", Nature 421 (6923), 611 (2003). 
[4] Nisoli M., De Silvestri S., and Svelto O., "Generation of high energy $10 \mathrm{fs}$ pulses by a new pulse compression technique", Appl. Phys. Lett 682793 (2006).

[5]Kling M. F., Siedschlag C., Verhoef A. J., Khan J. I., Schultze M., Uphues T., Ni Y., Uiberacker M., Drescher M., Krausz F. and Vrakking M. J. J., "Control of electron localization in molecular dissociation", Science 312 (5771), 246 (2006).

[6] Agostini P. and DiMauro L. F., “Atoms in high-intensity mid-infrared pulses”, Contemp. Physics 49 (3), 179 (2008).

[7]Mathias S., Wiesenmayer M., Deicke F., Ruffing A., Miaja-Avila L., Murnane M. M., Kapteyn H. C., Bauer M., and Aeschlimann M., "Time and angle resolved photoemission spectroscopy using femtosecond visible and high-harmonic light”, Journal of Physics: Conference Series 148012042 (2009).

[8] McKenna J., Bryan W. A., Calvert C. R., English E. M. L., Wood J., Murphy D. S., Turcu I. C. E., Smith J. M., Ertel K. G., Chekhlov O., Divall E. J., McCann J. F., Newell W. R. and Williams I. D. "Observing time-dependent vibrational quantum dynamics in deuterium hydride molecular ions", J Mod Opt 54 (7), 1127 (2007).

[9]Torres R., Siegel T., Brugnera L., Procino I., Underwood, Jonathan G., Altucci C., Velotta R., Springate E., Froud C., Turcu I. C. E., Ivanov M. Yu., Smirnova O., and Marangos J. P., "Extension of high harmonic molecular imaging by mid-IR laser fields", Optics Express 18, 3174-3180 (2010).

[10] Frassetto F., Bonora S., Villoresi P., Poletto L., Springate E., Froud C. A., Turcu I. C. E., Langley A. J., Wolff D. S., Collier J. L., Dhesi S. S., Cavalleri A., "Design and characterization of the XUV monochromator for ultrashort pulses at the ARTEMIS facility", SPIE Proc. Vol. 7077, Advances in X-Ray/EUV Optics and Components III, 707713 (2008).

[11] Frassetto F., Bonora S., Villoresi P., Poletto L., Springate E., Froud C. A., Turcu I. C. E., Langley A. J., Wolff D. S., Collier J. L., Dhesi S. S., Cavalleri A., "The XUV monochromator for ultrashort pulses at ARTEMIS", in Conference on Lasers and Electro-Optics 2009, OSA Technical Digest Series (Optical Society of America), JThE44 (2009).

[12] L. Poletto, P. Villoresi, F. Frassetto, "Diffraction gratings for the selection of ultrashort pulses in the extremeultraviolet", in "Advances in lasers and electro optics", V. Kordic (ed.), ITECHWEB, Wien (Austria), in print (2010).

[13] Bryan W. A., Stebbings S. L., McKenna J., English E. M. L., Suresh M., Wood J., Srigengan B., Turcu I. C. E., Smith J. M., Divall E. J., Hooker C. J., Langley A. J., Collier J. L., Williams I. D. and Newell W. R., “Atomic excitation during recollision-free ultrafast multi-electron tunnel ionization", Nature Physics 2, 379-383 (2006).

[14] Takahashi E. J., Hasegawa H., Nabekawa Y., and Midorikawa K., "High-throughput, high-damage-threshold broadband beam splitter for high-order harmonics in the extreme-ultraviolet region," Opt. Lett. 29, 507-509 (2004).

[15] Clerc F., Battaglia C., Bovet M., Despont L., Monney C., Cercellier H., Garnier M. G., Aebi P., Berger H. and Forró L., "Lattice-distortion-enhanced electron-phonon coupling and Fermi surface nesting in 1T-TaS,", Phys. Rev. B 74, 155114 (2006).

[16] Orr P. A., Williams I. D., Greenwood J. B., Turcu I. C. E., Bryan W. A., Pedregosa-Gutierrez J. and Walter C. W. Phys. Rev. Lett. 98163001 (2007)

[17] Alexander, J. D., Calvert, C. R., King, R. B., Kelly, O., Bryan, W. A., Nemeth, G. R. A. J., Newell, W. R., Froud, C. A., Turcu, I. C. E., Springate, E., Orr, P. A., Pedregosa-Gutierrez, J., Walter, C. W., Williams, R. A., Williams, I. D., Greenwood, J. B., "Short pulse laser-induced dissociation of vibrationally cold, trapped molecular ions", J. Phys. B 42 (15), 154027 (2009).

[18] Alexander, J. D., Calvert, C. R., King, R. B., Kelly, O., Graham, L., Bryan, W. A., Nemeth, G. R. A. J., Newell, W. R., Froud, C. A., Turcu, I. C. E., Springate, E., Williams, I. D., Greenwood, J. B., "Photodissociation of $\mathrm{D}_{3}^{+}$in an intense, femtosecond laser field", J. Phys. B 42 (14), 141004 (2009).

[19] Torres R., Kajumba N., Underwood, Jonathan G., Robinson J. S., Baker S., Tisch J.W. G., de Nalda R., Bryan W. A., Velotta R., Altucci C., Turcu I. C. E., and Marangos J. P., "Probing orbital structure of polyatomic molecules by high harmonic generation", Phys. Rev. Lett. 98, 203007/1-4 (2007).

[20] Kajumba N., Torres R., Underwood J. G., Robinson J. S., Baker S., Tisch J. W. G., de Nalda R., Bryan W. A., Velotta R., Altucci C., Procino I., Turcu I. C. E., and Marangos J. P., "Measurement of electronic structure from high harmonic generation", New J. Phys. 10, 025008/1-17 (2008).

[21] Torres R., Siegel T., Brugnera L., Procino I., Underwood Jonathan G., Altucci C., Velotta R., Springate E., Froud C., Turcu I. C. E., Patchkovskii S., Ivanov M. Yu., Smirnova O., and Marangos J. P., "Revealing molecular structure and dynamics through high harmonic generation driven by mid-IR fields", submitted to Physical Review Letters.

[22] Siegel T., Torres R., Hoffmann D. J., Brugnera L., Procino I., Zaïr A., J. G. Underwood J. G., Springate E., Turcu I. C. E., Chipperfield L. E., and Marangos J. P. "High harmonic emission from a superposition of multiple unrelated frequency fields", submitted to Optics Express. 